Franck DAMOUR, Stanislas DEPREZ et David DOAT, Généalogies et nature du transhumanisme. État actuel du débat

\title{
Gaël Brulé
}

\section{(2) OpenEdition Journals}

Édition électronique

URL : http://journals.openedition.org/ress/6189

DOI : $10.4000 /$ ress.6189

ISBN : 1663-4446

ISSN : $1663-4446$

Éditeur

Librairie Droz

Édition imprimée

Date de publication : 6 juillet 2020

Pagination : 285-288

ISSN : 0048-8046

Référence électronique

Gaël Brulé, « Franck DAMOUR, Stanislas DEPREZ et David DOAT, Généalogies et nature du

transhumanisme. État actuel du débat », Revue européenne des sciences sociales [En ligne], 58-1 | 2020, mis en ligne le 06 juillet 2020, consulté le 05 décembre 2020. URL : http://journals.openedition.org/ ress/6189; DOl : https://doi.org/10.4000/ress.6189

Ce document a été généré automatiquement le 5 décembre 2020

(C) Librairie Droz 


\title{
Franck DAMOUR, Stanislas DEPREZ et David DOAT, Généalogies et nature du transhumanisme. État actuel du débat
}

\author{
Gaël Brulé
}

\section{RÉFÉRENCE}

Franck DAMOUR, Stanislas DEPREZ et David DOAT (dir.), 2018, Généalogies et nature du transhumanisme. État actuel du débat, Montréal, Liber, 197 p.

1 Généalogies et nature du transhumanisme a pour but à la fois de montrer ce qu'est le transhumanisme et de susciter le débat en offrant une multitude de points de vue. À cette fin, onze chapitres regroupés en trois grandes parties (1. «Généalogie d'une idée »; 2. "Significations du transhumanisme » et 3. «Imaginaire et réalité ») se proposent de questionner les enjeux autour du transhumanisme, mouvement qui entend, pour le dire en des termes assez larges, augmenter l'homme par la technologie.

2 Il n'est jamais évident d'apprécier un ouvrage collectif : doit-on juger les parties qui le constituent, ce qu'elles recouvrent ou ce qu'elles laissent de côté ? Les angles abordés sont riches et l'on retrouve, à côté de chapitres descriptifs, des chapitres se positionnant pour et contre le transhumanisme avec des perspectives très différentes. Les coordinateurs ont fait le choix de donner un cadre souple au débat en réunissant une grande diversité d'auteurs. Si cette ouverture est louable et utile, il aurait été intéressant qu'ils les fassent dialoguer davantage et extraient les piliers constitutifs et les enjeux clivants du transhumanisme pour permettre au lecteur de se positionner. La nuance et la prudence sont salutaires mais, en indiquant dans le chapitre introductif que certains disent « ceci » et "d'autres cela », on a l'impression que les contributions « flottent » dans un débat sans cadre. 
3 On peut néanmoins tenter d'imaginer à quoi il aurait ressemblé. Tout d'abord, plutôt que de se demander si nous sommes pour ou contre le transhumanisme et d'empêcher le débat par des oppositions binaires, il semble plus intéressant de reconnaître que, pour nombre d'entre nous, nous acceptons un certain degré de transformation et d'amélioration par la technologie. Marc Roux (« Technoprogressisme et frontières de l'humain au-delà de l'horizon », p. 89-104), rappelle ainsi que la chirurgie ou le vaccin procèdent d'une certaine vision transhumaniste, et qu'une vaste majorité de femmes ont recours à l'avortement lorsqu'elles découvrent qu'elles portent un enfant trisomique, une forme d'eugénisme constitutif de la pensée transhumaniste. La plupart d'entre nous se retrouveraient ainsi dans certaines propositions issues de la déclaration transhumaniste : « on devrait mener des recherches méthodiques pour comprendre ces futurs changements [de la technologie] mais aussi leurs conséquences à long terme » ou encore «nous devons créer des forums où les gens pourront débattre en toute rationalité de ce qui devrait être fait mais aussi d'un ordre social où on puisse mettre en œuvre des décisions responsables» (voir <https://iatranshumanisme.com/ transhumanisme/la-declaration-transhumaniste>).

4 Mais reconnaître un certain degré de transformation ne veut pas pour autant dire "puisque nous y sommes déjà, autant y aller à fond ", comme le voudrait un procédé rhétorique de certains enthousiastes du mouvement; nous serions choqués par une augmentation sans complexe de l'être humain qui aboutirait à une humanité à deux vitesses. Si aucun auteur ne revendique cette intention, le libertarianisme assumé des élites culturelles, économiques et technologiques couplé à la montée des inégalités actuelles semble y mener tout droit. La plupart des adeptes du libre-échange n'ont jamais revendiqué une volonté explicite de créer des inégalités fortes, mais les choix effectués y conduisent de facto. Avant même de se positionner pour ou contre le transhumanisme ou même sur un continuum allant de " pas du tout favorable » à « tout à fait favorable ", il semble nécessaire de mettre en avant deux apories majeures de l'épistémè transhumaniste actuelle: la question socio-politique et la question écologique.

5 Les coordinateurs du livre défendent une vague adhésion à la déclaration transhumaniste: "les transhumanistes prônent le droit moral de ceux qui le désirent de se servir de la technologie pour accroître leurs capacités physiques, mentales ou reproductives et d'être davantage maîtres de leur propre vie » (ibid.). Mais revendiquer une utilisation isolée de la technologie est à peu près aussi crédible que de dire «je prône le droit de jouer avec la bombe atomique dans mon jardin sans que celui d'à côté ne s'en émeuve ». Tout acte, toute intention, porte en lui une vision du monde. En choisissant d'acheter tel ou tel produit, d'être propriétaire d'une maison, ou d'investir dans le cacao en Côte d'Ivoire, je pousse une vision de la société, mon action est de fait politique. Dès lors, avoir des humains augmentés n'est pas sans conséquence pour le voisin qui ne l'est pas. Francesco Paolo Adorno («L'illusion de la liberté. Transhumanisme et biopolitique», p. 105-118) rappelle que la politique est engagée dans la réalisation de ces finalités. Ce dernier voit dans le mouvement transhumaniste un effet libérateur qui relève de l'hallucination. Comme le rappelle Benjamin Bourcier (« La politique transhumaniste : pari utilitariste et politique du futur?», p. 179-192), le libertarianisme affiché de la plupart des grands noms du transhumanisme semble d'une naïveté étonnante. Celle-ci renvoie à des individus éblouis et non éclairés pas la technologie. 
6 La légitimation du mouvement par les adeptes est faite en traçant des filiations étymologiques ou épistémologiques qui n'existent pas forcément. Jean-Yves Goffi ("L'héritage de la génération Huxley-Teilhard de Chardin», p. 35-54) rappelle que l'émergence du terme de transhumanisme est intéressante puisqu'elle procéderait d'une nouvelle de Julian Huxley, qui n'aurait pas été des plus enthousiastes à l'égard de son néologisme et lui aurait préféré pendant longtemps le terme d'humanisme évolutionnaire. Ce sont les transhumanistes des générations suivantes, comme Nick Bostrom, qui auraient voulu figer ce terme. Nicolas Le Dévedec («Humanisme, transhumanismes: deux conceptions antithétiques de la perfectibilité humaine», p. 19-34) récuse le rapprochement communément fait entre humanisme et transhumanisme, puisque de nombreux transhumanistes se voient comme héritiers des Lumières, et notamment de figures comme le Marquis de Condorcet. Or, s'il y a bien une revendication du progrès au sein du mouvement large des Lumières, jamais il ne s'y réduit ; la technique est un outil pour l'autonomie politique (p. 22). Cette dimension politique est absente de la pensée transhumaniste, qui ne s'intéresse en général qu'à l'individu. Les transhumanistes autonomisent le progrès comme quelque chose de désirable en soi et pour soi. Selon Marc Roux, leur mouvement a tendance à délaisser l'impact social du transhumanisme, avec un risque clair: que les avancées technologiques ne bénéficient qu'aux plus riches, et que l'on aille vers une humanité à deux vitesses. L'éthos transhumaniste est néolibéral et les transhumanistes comme Ray Kurzweil, cité dans pratiquement chaque chapitre du livre, semblent préférer la science à la vie. Celles et ceux qui pensent que la technologie répondra à tous les problèmes, y compris à ses propres apories, paraissent faire preuve d'une cécité historique. Il n'est pas possible de regarder le transhumanisme seulement à travers le prisme technologique, il convient de le regarder à travers le prisme social et politique, comme le dit justement Nicolas Le Dévedec dans son chapitre. L'angle politique est trop souvent soit omis, soit rejeté.

7 Si la pensée écologique est abordée dans le chapitre de Thierry Magnin et Sylvie Allouche par la voix du pape François («Le transhumain et l'anthropologie chrétienne », p. 129-146), un réel point de vue écologique aurait été salutaire pour éclairer la question du transhumanisme. Le transhumanisme ne se dilue pas aisément dans la pensée écologique, ou alors sous des formes extrêmes (on peut penser au nazisme qui combinait les deux). Une vraie démarche écologique s'appuie sur le principe de précaution, qui consiste à ne pas faire des choses dont on maîtriserait mal les conséquences. Le mouvement transhumaniste rejette ce principe et lui préfère le principe d'extropie, (un néologisme construit en opposition au principe entropique et à l'idée d'une inévitable dégradation de la matière et de la vie). Marc Roux le dit même directement : " plutôt que de révérer la finitude, attitude qui se traduit parfois par des tendances morbides ou nihilistes, le transhumanisme appelle à ouvrir nos sociétés à d'autres horizons » (p. 99). Par exemple, « la venue au monde d'une personne humaine d'origine animale pourrait être une formidable source d'enrichissement " (p. 97). Les transhumanistes autonomisent la technologie, qui était autrefois un medium et semblent ne poser que très rarement la question du pourquoi. Gilbert Hottois (« Pour un transhumanisme philosophique critique», p.73-88) semble prôner un transhumanisme plus modéré et plus fin. S'appuyant sur une expérience de la bioéthique en comité, et en considérant le transhumanisme en tant que matérialisme non-métaphysique, il permet de s'extraire des pensées utopiques ou dystopiques pour aller sur le terrain de la discussion et de l'échange sur les conséquences, les coûts, etc. 
de l'adoption d'une technologie biomédicale. Chaque avancée doit se faire de manière débattue. Encore faudrait-il que la société civile soit bien représentée au sein de ces comités et que sa voix porte, au moment de l'adoption de telles technologies, pour la prise en compte des effets sociaux et environnementaux. Selon Marc Roux, le transhumanisme se propose d'" oser franchir le seuil» et «de laisser courir notre curiosité vers d'autres horizons, car sans curiosité, il n'y a pas d'avenir possible » (p.93). D'un point de vue écologique, on pourrait dire que cette curiosité ressemble beaucoup à celles qui a légitimé la surexploitation de la terre depuis des décennies, et hypothéqué l'avenir de la planète. On se rapprochera donc plutôt de la critique de Nicolas Le Dévedec, qui marque bien les aspects politiques souvent oubliés par les enthousiastes du transhumanisme, et de Thierry Magnin et Sylvie Allouche, lesquels préfèrent à la simplicité des solutions techniques la complexité du vivant.

Tels qu'ils sont définis et tels qu'ils de définissent actuellement, les transhumanistes semblent être le résultat de passionnés de technologie et d'évangélistes. Revendiquant le matérialisme et le rejet des croyances, ils se placent pourtant, avec leurs aspirations immortalistes, dans un registre liturgique et fétichiste de la technologie et de l'intelligence artificielle (voir les chapitres de Paul Jorion «Les préconisations du transhumanisme sous le regard de l'anthropologue et de l'éthologue », p. 119-128, et de Salomé Bour «Mythologies transhumanistes », p. 147-162). L'univers parfait cartésien recherché par certains technologistes reprend point par point les symboles religieux. Selon Cecilia Calheiros ("Aspirations métaphysiques et attentes eschatologiques chez les transhumanistes ", 2019, Revue d'éthique et de théologie morale, 302-2, p. 43-57), «le transhumanisme qui propose le remplacement de la religion par la rationalité scientifique finit, par symétrie, par prétendre aux mêmes vertus et à reproduire les mêmes rhétoriques» (p.56). Selon Miguel Benasayag, le transhumanisme porte en lui la « haine du corps et de la vie » et une posture politique réactionnaire (entretien avec Miguel Benasayag, Reporterre, 6 mai 2017 : <https://reporterre.net/Miguel-BenasayagLe-transhumanisme-prepare-un-monde-d-apartheid>). Certains sujets sont si importants qu'ils nécessitent que l'on se positionne par rapport à eux. On aurait aimé que les directeurs du livre mettent davantage en relief certaines apories évidentes (sociales, politiques, écologiques) du transhumanisme tel qu'il est évoqué actuellement. Peut-être sera-ce l'occasion d'un futur ouvrage?

\section{AUTEURS}

\section{GAËL BRULÉ}

Université de Genève 